\title{
Clinical utility of vilazodone for the treatment of adults with major depressive disorder and theoretical implications for future clinical use
}

This article was published in the following Dove Press journal:

Neuropsychiatric Disease and Treatment

21 March 2012

Number of times this article has been viewed

\section{Mandeep Singh \\ Thomas L Schwartz \\ SUNY Upstate Medical University, Psychiatry Department, Syracuse,} NY, USA
Correspondence: Thomas L Schwartz SUNY Upstate Medical University, Psychiatry Department, 750 East Adams Street, Syracuse, NY 13210, USA

$\mathrm{Tel}+\mathrm{I} 3154643166$

Fax + I 3I54643I63

Emailschwartt@upstate.edu
Background: Vilazodone is the latest approved antidepressant available in the United States. Its dual mechanism of action combines the inhibition of serotonin transporters while simultaneously partially agonizing serotonin-1a (5-HT1A) receptors. This combined activity results in serotonin facilitation across the brain's serotonergic pathways, which has been termed by the authors as that of a serotonin partial agonist and reuptake inhibitor, or SPARI.

Objective: The authors to review laboratory, animal model data, and human trial data to synthesize a working theory regarding the mechanism of antidepressant action of this agent and regarding its potential for additional indications.

Methods: A MEDLINE and Internet search was conducted and the resultant evidence reviewed.

Results: Vilazodone has randomized, controlled empirical data which has garnered it an approval for treating major depressive disorder. It combines two well-known pharmacodynamic mechanisms of serotonergic action into a novel agent. Although no head-to-head studies against other antidepressants are published, the efficacy data for vilazodone appears comparable to other known antidepressants, with associated gastrointestinal side effects similar to serotonin selective reuptake inhibitor and serotonin norepinephrine reuptake inhibitor antidepressants, but potentially with a lower incidence of sexual side effects and weight gain.

Discussion: As a new option for the treatment of major depressive disorder, vilazodone, due to its unique SPARI mechanism of action, may hold promise for patients who cannot tolerate or have not responded to previous antidepressant monotherapies. Additionally, its use may extend to the treatment of other mental health conditions similar to those treated by serotonin selective reuptake inhibitors.

Keywords: major depressive disorder, vilazodone, antidepressants

\section{Introduction}

Vilazodone is now a US Food and Drug Administration (FDA)-approved antidepressant treatment (ADT). This paper will review preclinical pharmacokinetic information, pharmacodynamic information, current publicly available clinical data for this product, as well as reviewing animal models and mechanism of action data that would suggest potential use in other realms of psychiatric illness.

Clinicians have been using the same catecholamine treatments for major depressive disorder (MDD) since the 1950s, mostly by blocking reuptake transporters (dopamine, norepinephrine, and serotonin). Originally utilizing the tricyclic antidepressants, and in recent years, more so with serotonin selective reuptake inhibitors (SSRIs) and serotonin norepinephrine reuptake inhibitor (SNRIs). The National Institute of 
Mental Health's STAR*D trial suggests that remission from a prototypical agent of the SSRI class occurs one-third of the time with initial monotherapy in MDD patients, and each subsequent ADT yields less favorable outcomes as treatment resistant depression increases. After four successive ADTs, about two thirds of patients finally remit, but many of these do not sustain their remission for more than a few months. ${ }^{1}$ Therefore, one third of MDD patients continue to have significant symptoms after treatment with a sequence of agents for about a year, and many of those who remit do not sustain their improvement. Given these modest results, researchers continue to investigate ways to treat MDD with novel pharmacologic mechanisms.

In the absence of a remarkable breakthrough drug in the area of nonmonoamine agents, ie, hormonal, peptide, genetic, neuromodulation, ${ }^{2}$ clinicians have resorted to higher levels of polypharmacy to gain full remission when monotherapies fail. Combination drug treatment might be being deployed earlier and earlier in treatment as an option. ${ }^{1,3,4}$ To boost antidepressant efficacy in patients who fail to respond adequately to a SSRI, second generation atypical antipsychotics are FDA-approved (aripiprazole, quetiapine, quetiapine $\mathrm{XR}$, olanzapine-fluoxetine combination), but with potential additional side effect burden (metabolic and movement disorders) and cost. ${ }^{5,6} \mathrm{~A}$ unique mechanistic approach occurs with vilazodone, an agent that combines two mechanisms in a single drug, namely that of a SSRI with 5-HT1A receptor partial agonist actions serotonin partial agonist reuptake inhibitor (SPARI). ${ }^{7}$ Specifically, this agent increases the availability and activity of the neurotransmitter serotonin and its neuropathways. Vilazodone blocks the serotonin reuptake pump (serotonin transporter), desensitizes serotonin receptors (especially serotonin 1A autoreceptors), and therefore presumably increases serotonergic neurotransmission. Its partial agonist actions at presynaptic somatodendritic 5-HT1A autoreceptors may theoretically enhance serotonergic activity and contribute to antidepressant actions as well. ${ }^{1,8,9}$ This partial agonist action also occurs at the level of the postsynaptic 5-HT1A receptor which may theoretically diminish sexual dysfunction. ${ }^{8,9}$ This effect has been noted in studies utilizing the 5-HT1A receptor partial agonist, buspirone. ${ }^{10}$ In support of this theoretical information, similar animal models suggest potential for rapid onset antidepressant efficacy, given more robust serotonergic actions suggesting greater antidepressant efficacy compared to SSRIs. ${ }^{11-13}$ However, these preclinical suggestions have yet to be confirmed specifically for vilazodone in human clinical trials. Currently there is a lack of head-to-head comparative trials with other antidepressants.
This makes potential efficacy and tolerability comparisons to known ADT agents difficult. What is known about the pharmacokinetics, pharmacodynamics, and clinical trial results of vilazodone and animal models could suggest further applications for this novel mechanism. This information will be reviewed shortly.

\section{Vilazodone pharmacokinetics}

According to the official FDA-sanctioned package insert, ${ }^{14}$ this drug is initially dosed at $10 \mathrm{mg}$ per day in the morning for 1 week then dose escalated to $20 \mathrm{mg}$ per day for week 2 with the final titration to the $40 \mathrm{mg}$ per day as a usual daily dose. It comes in $10 \mathrm{mg}, 20 \mathrm{mg}$, and $40 \mathrm{mg}$ tablet strengths. This drug must be taken with food or $50 \%$ of its bioavailability is lost. There are no dosing changes required in renal or hepatic patients and a gradual withdrawal is suggested to avoid serotonin discontinuation syndrome. Its moderate half-life makes withdrawal possible, but likely with a low severity of symptoms. It is clinically contraindicated for use with monoamine oxidase inhibitors. Vilazodone is metabolized extensively by the hepatic CYP450 3A4 enzyme system. Its dose should be reduced to $20 \mathrm{mg} /$ day with concomitant use of potent 3A4 inhibitors (erythromycin, amiodarone, protease inhibitors, ketoconazole). Vilazodone's therapeutic activity is due primarily to the parent drug and there are no known active metabolites. The pharmacokinetics of vilazodone ( $5 \mathrm{mg}-80 \mathrm{mg}$ ) are dose-proportional. Steady-state is often achieved in days. Vilazodone has a terminal half-life of approximately 25 hours. It is 96\%-99\% protein-bound so it may disrupt digoxin- or coumadin-binding temporarily as it displaces these drugs into a nonprotein-bound, free plasma state which increases their availability and activity.

\section{Vilazodone pharmacodynamics}

Vilazodone is a combined serotonin reuptake inhibitor (SRI) and 5-HT1A receptor partial agonist. ${ }^{15}$ The authors use the term SPARI to define this class of $\mathrm{ADT}^{7}$ This mechanistic way of treating MDD is similar to the common depression treatment strategy of augmenting SSRI monotherapy (fluoxetine, sertraline, paroxetine, etc) with the commercially available 5-HT1A receptor partial agonist anxiolytic, buspirone. ${ }^{16}$ The latter is approved for treating generalized anxiety disorder (GAD). ${ }^{7}$ In fact, the STAR*D trial has studied nonremitters to treatment with citalopram, comparing augmentation with either buspirone or with bupropion SR, finding no significant differences in remission rates between these two combination treatments. ${ }^{17}$ 
SRI mechanisms yield an increase in synaptic 5-HT through serotonin transporter reuptake inhibition. This produces a desensitization and/or downregulation of presynaptic 5-HT1A autoreceptors. As these autoreceptors are now over stimulated, the 5-HT neuron interprets this initially as toxic activity. As these autoreceptors become less effective due to over stimulation and desensitization, they offer less autoreceptor inhibition to the 5-HT neuron and excess 5-HT is next released at the neuron terminals as a result. By directly agonizing the 5-HT1A receptors in the central nervous system, vilazodone likely allows a faster or greater volume of 5-HT1A receptor desensitization/downregulation up front. This may lend more additive or synergistic 5-HT effects than SSRI alone. Thus, the rationale behind this pharmacodynamic combination is to yield a faster and larger scale desensitization of the somatodendritic 5-HT1A autoreceptor system in MDD patients. This mechanism essentially lowers the 5-HT neurons' ability to regulate 5-HT output, thus increasing 5-HT neuronal firing and activity. This more aggressive, or synergistic, 5-HT facilitation approach may then directly stimulate postsynaptic 5-HT1A heteroreceptors downstream which may actually promote even more neuronal firing and activity of 5-HT or other monoamine neurons. ${ }^{13}$

The recent review by Athanasiou, Reed, and Rickels suggests that in vitro studies show that vilazodone is more highly selective for the 5-HT1A receptor than for other neuronal receptors and its affinity $(50 \%$ inhibitory concentration $\left.\left[\mathrm{IC}_{50}\right] \mathrm{nM}\right)$ is 0.5 compared to a lower affinity buspirone anxiolytic. ${ }^{18}$ The 5-HT1A receptors are presynaptic receptors located in the raphe nuclei where they act as autoreceptors to inhibit the firing rate of 5-HT neurons. The 5-HT1A receptors are also located on postsynaptic cells in limbic and cortical regions where they can also lower firing.

Although this theoretical thinking underlies the development of vilazodone as a potentially novel antidepressant drug, there is as yet no clinical human evidence of rapid onset or more robust antidepressant action compared to SSRI/SNRI antidepressants. The presence of gastrointestinal side effects causes the need for slower titration of vilazodone starting at lower than the maintenance dosing for 2 weeks, thus potentially delaying any potential rapid onset effect in patients for better tolerability.

As there are limited animal models and no direct vilazodone head-to-head comparative trials available, a single, monotherapy agent like vilazodone that combines these same pharmacologic actions as the combination of a SSRI with buspirone should be able to provide the potential efficacy benefits of this combination, particularly if administered early in treatment. ${ }^{19,20}$ Instead of starting with a SSRI monotherapy with dose escalation for 12 weeks, waiting for it to potentially fail (two of three times per STAR $* D$ ) followed by the subsequent augmentation addition of buspirone for another several weeks, vilazodone allows immediate, simultaneous combination of these two pharmacodynamic properties at the outset of treatment. Athanasiou et al reviewed the pharmacogenetics associated with vilazodone which in theory might allow clinicians to genetically test which patients might respond more fully to vilazodone and perhaps detect who will experience adverse side effects. Regarding gastrointestinal side effects, genetic biomarkers (M2-) may suggest those with a 10-fold increase in risk of severe gastrointestinal distress. Finally, patients with M1+ genetic markers are more likely to have full antidepressants effect towards remission with a relative risk of 2.1. ${ }^{18}$

Overall, this product is not as complex or risk-laden as a second generation atypical antipsychotics augmentation approach in treating MDD, it would in theory allow less side effect burden, especially given its absence of metabolic and movement disorders. A full discussion of the clinical effectiveness and adverse effects of vilazodone are forthcoming. Unfortunately, these considerations and comparisons to STAR $*$ D outcomes remain merely theoretical since there are no studies reporting the comparison of vilazodone to other active treatments or combinations of treatments.

\section{Serotonin and brain circuitry: a primer}

This circuitry is reviewed to indicate where vilazodone may exert its effects and also to alert the reader to better understand the forthcoming discussion regarding vilazodone's preclinical data.

Serotonergic pathway projections include the spinal cord, brainstem cortical, and subcortical structures. The 5-HT system originates from neurons of the brainstem raphe nuclei and project widely throughout the brain to regulate many functions, including sleep, mood, and stress reactivity. These are implicated in the pathogenicity of MDD and anxiety disorders. ${ }^{21}$

The ascending serotonergic pathways project from the raphe to the amygdala, the paralimbic entorhinal and dentate gyrus, the rectus gyrus of the frontal lobe, and the inferior and superior gyri of the temporal lobe. Multiple 5-HT receptors have been implicated including 5-HT1A, and 5-HT1B/5-HT1C which are found in different areas of the brain that interact with the hypothalamic-pituitary-adrenal (HPA) axis. In particular 5-HT1A receptors in man, rat, 
and guinea pig are found in high concentration in the dorsal raphe nuclei (presynaptic), the hippocampus (postsynaptic), the lateral septum, the frontal cortex, and the dorsal horn of the spinal cord. ${ }^{21}$

Evidence has shown a lower density of 5-HT1A receptor sites in the amygdala and hippocampus in depressed patients which may be related to the increased HPA activity consistent with increased glucocorticoid secretion found in these patients. This increased activity of the HPA axis is related to increased corticotrophin-releasing hormone, potentiating effects of increased arginine vasopressin secretion on corticotrophin-releasing hormone-induced ATCH secretion which may be due to the diminished central 5-HT neurotransmission that allows for the negative feedback of this system. ${ }^{20}$ Therefore, not only does serotonergic dysfunction itself play a role in MDD and anxiety disorders, but the interplay with the HPA axis and 5-HT1A receptors are of special interest.

\section{Vilazodone in animal studies}

The role of 5-HT in regards to its anxiolytic effect has been illustrated in rodent distress vocalization studies in the expression of ultrasonic calls of infant rat pups during brief maternal separations. ${ }^{21}$ In this model there was a reduction in the rate of calling after acute administration of SSRIs to 9-11-day-old pups. Similarly, acute administration of the 5-HT1A agonists buspirone, 8-OH-DPAT and ipsapirone reduced the rate of calling at doses that did not affect motor activity or core body temperature. ${ }^{22}$ In similar ultrasonic vocalizations studies using vilazodone, stressinduced vocalizations were inhibited as well $(55 \mathrm{mg} / \mathrm{kg}$ po) at 120 minutes and 210 minutes post-dose. In contrast, the SSRI fluoxetine (100 mg/kg po) was without effect unless combined with 8-OH-DPAT. This suggests that the 5-HT1A receptor agonist activity in vilazodone contributed immensely to its efficacy. ${ }^{23}$

In two other rat study models for anxiety, vilazodone demonstrated dose-related efficacy (10-40 mg/kg ip) in the shock probe test ${ }^{24}$ which measures the defensive burying behavior by a rat in response to anxiety provoking stressors (a shock by stationary electrified probe), but no significant effect was found in the elevated plus maze where the animal is placed in the center of an elevated four-arm maze (two arms which are open and well lit and two arms that are enclosed and dimly lit). The time the rat spends on each arm is recorded. Rats usually prefer the closed arms but when effective anxiolytics are administered they increasingly enter the open arms with more propensity. ${ }^{23,25}$
Vilazodone was also examined in a predator-induced stress paradigm, when exposure to a domestic cat would result in additional increased anxiety-like behaviors in the elevated plus maze and an increased response to an acoustic startle. Diminished stress-induced potentiated startle were observed when vilazodone (20-40 mg/kg ip) was administered acutely or prophylactically (1 week prior to behavioral testing), but had no effect on stress potentiated anxiety response in the elevated plus maze. ${ }^{26}$

In studying vilazodone antidepressant-like activity, two instances using the forced swim test, which are often used to screen for effective antidepressants, revealed some support for this effect. Rats who were injected with three doses of vilazodone ( $1 \mathrm{mg}, 3 \mathrm{mg}$, and $10 \mathrm{mg} / \mathrm{kg}$ ) prior to the swim test showed reduced immobility and increased swimming behavior although only significant changes were observed at the $1 \mathrm{mg} / \mathrm{kg}$ dose. Swimming was defined as horizontal movement through a swim chamber which included crossing into another quadrant in a 5-minute swim test after being placed in a cylinder full of water for 15 minutes. In the mouse forced swim test similar results were produced at the $1 \mathrm{mg} / \mathrm{kg}$ dose, however, no significant changes were observed at $0.3 \mathrm{mg} / \mathrm{kg}$ and $3 \mathrm{mg} / \mathrm{kg}$ doses. These are consistent with patterns observed with conventional SSRIs such as fluoxetine in the past. ${ }^{27,28}$

In vitro tests demonstrated that vilazodone has serotonin neurotransmission enhancing potential in part due to robust blockade of the serotonin transporter and in part to direct partial agonist actions on 5-HT1A receptors. ${ }^{27}$ In fact, one such study revealed that vilazodone increased cortical 5-HT levels more than a SSRI (fluoxetine) alone, making intuitive sense in two serotonergic mechanisms, which should have additive cortical effects. In the rat cerebral cortex vilazodone inhibited serotonin reuptake 30 times more than fluoxetine. ${ }^{29}$ Alternatively, one study revealed that vilazodone behaved essentially as a SSRI in the guinea pig dorsal raphe, using voltammetric measures of 5-HT, but displayed no evidence of direct agonism at 5-HT1A receptors, ${ }^{27}$ fostering controversy over 5-HT additive or synergistic effects in animal models.

\section{Vilazodone in human studies}

In a double-blind placebo-controlled crossover in vivo study with ten healthy males, the effect of vilazodone at $20 \mathrm{mg}$ and $40 \mathrm{mg}$ was assessed on sleep electroencephalography, suggesting that at the $40 \mathrm{mg}$ dose it preferentially occupied presynaptic versus postsynaptic 5-HT1A receptors. ${ }^{27}$

Although in vivo data has shown some inconsistencies in its action as an agonist at pre-and/or postsynaptic 
5-HT1A receptors, vilazodone does enhance forebrain serotonergic output and is efficacious in preclinical models of anxiety and depression regardless. ${ }^{13}$

Taking into account studies showing partial agonism at the 5-HT1A receptors increasing serotonin levels in brain areas relevant to depression and the synergy achieved in combination with a SSRI, vilazodone's 5-HT effect may be more pronounced due to additional data suggesting that it is 60 times more selective for the 5-HT1A receptor compared with buspirone with an $\mathrm{IC}_{50}$ of $0.5 \mathrm{nM}$ versus $30 \mathrm{nM}$ for buspirone. Vilazodone is also approximately 30 times more selective at blocking serotonin reuptake compared to fluoxetine with an $\mathrm{IC}_{50}$ of $0.2 \mathrm{nM}$ versus $6 \mathrm{nM}^{28}$

In vivo vilazodone was more potent than fluoxetine at inhibiting serotonin reuptake in mouse hypothalamic synaptosomes. ${ }^{29}$

Initial human studies showed sleep architecture changes consistent with other approved antidepressants..$^{30}$ Dawson's ${ }^{13}$ review outlines phase II studies in a succinct manner where vilazodone was administered to a total of 369 healthy subjects and 1163 depressed subjects, but failed to demonstrate significant efficacy against placebo initially. Of note, active comparator studies here also failed to demonstrate efficacy versus placebo. However, subsequent clinical trials have demonstrated statistical significance against placebo in phase III trials for MDD. ${ }^{31}$ This suggests some controversy where some studies were positive and some negative. The more current, larger scale studies with improved statistical power and design have suggested enough separation from placebo to allow FDA approval. Vilazodone was well tolerated, with the most commonly observed adverse events being diarrhea, nausea, somnolence, and dizziness. An FDA new drug application occurred in 2009 and secondary, regulatory trials again suggested antidepressant efficacy and tolerability. Vilazodone was approved for MDD in January 2011.

The official company data and FDA package insert ${ }^{14}$ reveals this positive regulatory confirmatory trial information. Vilazodone was effective in two pivotal short-term studies, double-blind phase III studies both 8 weeks in length.

One a randomized, controlled trial where 410 subjects aged 18-65 years found significant improvements in multiple depression rating scales when compared to placebo. Effectiveness was assessed with the Montgomery-Asberg Depression Rating Scale and clinical global impressions scales as well as with the 17-item Hamilton Rating Scale. Diarrhea, nausea, and somnolence were the greatest side effects noted. There were clinically no significant differences for either gender in regards to sexual dysfunction outcome measures. ${ }^{32}$ Vilazodone efficacy in MDD was further established in 8-week, multicenter, randomized, doubleblind, placebo-controlled studies in adult (18-70 years of age) outpatients who met the Diagnostic and Statistical

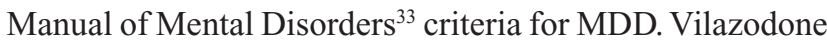
was superior to placebo in the improvement of depressive symptoms, and evaluation of population subgroups based on age, gender, and race did not reveal any clear evidence of differential responsiveness. Its most common side effects over placebo included diarrhea, nausea, and dry mouth and does carry the increased lethality warning in young adult patients. It is not approved for pediatric depression. No blood laboratory or EKG changes were noted and none are required while administering this ADT. In these acute 8-week studies no abnormal weight gain or metabolic adverse effects were noted. ${ }^{14}$ Finally, sexual dysfunction adverse effects were minimal ( $1 \%-2 \%$ over placebo rates only). ${ }^{7}$ Of course, minimal side effects in these areas still suggest that they may occur and some studies show better tolerability than others.

Additional phase II studies were all 8 weeks and used the Hamilton Rating Scale as primary endpoints while using the Montgomery-Asberg Depression Rating Scale and clinical global impressions as secondary endpoints. Reviews of previous studies showed significant secondary endpoint improvement in clinical global impressions severity score as did a second placebo controlled study using fixed dose scheme while again no significant differences were found between placebo and treatment for primary outcomes. ${ }^{31}$

In two more phase II studies, vilazodone and fluoxetine at typical starting doses were compared against placebo, no significant difference was found versus placebo in primary outcomes but were statistically significant in secondary outcomes. $^{31}$

In regard to long term studies, a 52 week open label study including 616 patients aged 18-70 years found improvement in Montgomery-Asberg Depression Rating Scale scores over this time period. They concluded vilazodone $40 \mathrm{mg}$ /day for 1 year was safe and well tolerated by adults with MDD. ${ }^{34}$

Additionally, a review of data from 24 human trials which included 2898 human subjects exposed to one or more doses of vilazodone concluded that it was as effective as SSRIs, however, stated it to be unknown if it had any advantages over others in its class. ${ }^{35}$

\section{Other clinical applications}

Given that other agents with SSRI activity and 5-HT1A receptor activity carry approvals for depression, GAD, 
obsessive compulsive disorder (OCD), social anxiety disorder (SAD), panic disorder, posttraumatic stress disorder, eating disorders, and premenstrual disorders, ${ }^{7,36}$ it would follow that vilazodone conceptually has the pharmacodynamic mechanisms necessary to alleviate symptoms attributed to these disorders as well.

\section{GAD}

Currently buspirone is FDA-approved for treatment of GAD. Studies have shown similar effects compared to various benzodiazepines without dependence and discontinuation issues ${ }^{22}$ which are a benefit especially in specific populations with substance abuse history or the elderly. By extension of this similar mechanism of action, vilazodone could be a feasible option in treatment resistant GAD and given the $50 \%$ comorbidity with MDD, would be beneficial in these cases as well, due to its antidepressant effect.

\section{Alcohol abuse}

Studies using buspirone have been conducted and shown to reduce anxiety and cravings in patients with alcohol dependence. ${ }^{22}$ In a placebo-controlled double-blind trial involving 51 subjects, buspirone was superior to placebo in reducing the number of days craving alcohol. This is a potentially advantageous use for vilazodone due to the common comorbid diagnosis of depression with alcohol dependence in one third of these patients. ${ }^{37}$

\section{OCD}

A small open-label study ${ }^{38}$ found some benefit from nine out of eleven patients with OCD when buspirone was added to fluoxetine which replicates the SPARI mechanism of vilazodone. However, a subsequent placebo-controlled double-blind study did not show an advantage over placebo in similar augmentation of bupsirone. ${ }^{39}$ Therefore, although there may be a role for 5-HT1A agonism in OCD treatment more studies are required to fully explore this combination treatment as a viable option. It is likely that vilazodone doses would need to be utilized greater than $40 \mathrm{mg} /$ day, similar to the excessive SSRI doses needed to treat OCD.

\section{SAD}

In a study ${ }^{40}$ with 21 social phobia patients, $47 \%$ showed modest improvement when using buspirone in an open 12-week trial. Comparable results were shown in another open buspirone trial ${ }^{26}$ which could suggest a role for vilazodone for the treatment of SAD with its built-in 5-HT1A partial agonist mechanism.
Also, since $40 \mathrm{mg}$ only occupies an estimated $50 \%$ of serotonin transporters and 5-HT1A receptors, ${ }^{41}$ it seems reasonable to test doses in the $50-80 \mathrm{mg}$ /day range by slow upward titration, especially for treatment resistant cases of depression and other related disorders.

\section{Conclusion}

Vilazodone has been approved for treatment of MDD. Usual treatment guidelines ${ }^{42}$ should be followed in regards to making an accurate diagnosis, ruling out bipolarity, substance misuse, and personality disorders prior to its use. If an ADT is warranted, monotherapy with an approved agent with a good risk/benefit should be chosen. Vilazodone should be acceptable as a first-line agent as its combined SPARI mechanism offers a unique initial antidepressant approach when compared to SSRI, SNRI, etc. The dose of vilazodone must be more fully explored as well, because a clear "no effect" dose has not been established and a $20 \mathrm{mg}$ dose trial will be required as a condition of approval, as will studies in children and longer term relapse prevention studies in depression. Vilazodone, however, will likely be used in those who do not respond to a SSRI/SNRI or do not tolerate a SSRI/SNRI given their prevalence and ease of use as well as potential for faster titration and lower gastrointestinal side effect profiles. Vilazodone may be especially useful if the patient develops sexual dysfunction, weight gain, or increased blood pressure on these agents, and should strongly be considered secondarily, if patients cannot tolerate or risk intervention with an atypical second generation antipsychotic augmentation trial due to weight gain, sedation, extrapyramidal symptoms, or dyslipidemia. Additionally, in examination of its mechanism of action being similar to SSRIs plus buspirone, as well as results from its animal model studies, its clinical application can be theoretically broadened off label for the treatment of other psychiatric disorders, especially if initial approved drug trials fail.

In regards to future investigations, given this products dual action manipulation of serotonergic processes it should undergo extensive clinical trial investigation for the treatment of SAD, panic disorder, $\mathrm{OCD}$, posttraumatic stress disorder, bulimia nervosa, and premenstrual dysphoric disorder comparable to those indications held by current SSRI and SNRI antidepressant products.

\section{Acknowledgments}

The authors would like to thank Dr. Stephen Stahl for his mentorship regarding translational concepts while creating this manuscript. 


\section{Disclosure}

The preparation of this review was not supported by any external funding. The authors report no conflicts of interest in this work.

\section{References}

1. Rush AJ, Triveda MH, Wisniewski SR, et al. Acute and longer-term outcomes in depressed outpatients requiring one or several treatment steps: a STAR*D report. Am J Psychiatry. 2006;163(11): 1905-1917.

2. Schwartz TL, Stahl SM. Optimizing antidepressant management of depression: current status and future perspectives. In: Cryan JF, Leonard BE, editors. Depression: From Psychopathology to Pharmacotherapy. Basel, Switzerland: Karger; 2010;27:254-267.

3. Schwartz TL, Rashid A. Augmentation and combination pharmacotherapy trends in major depressive disorder: results of a brief survey of psychiatrists. J Clin Pharm Ther. 2007;32(1):28-31.

4. Blier P, Ward HE, Tremblay P, Laberge L, Hébert C, Bergeron R. Combination of antidepressant medications from treatment initiation for major depressive disorder: a double-blind randomized study. Am J Psychiatry. 2010;167(3):281-288.

5. Papakostas GI. Use of atypical antipsychotics as augmentation for treatment-resistant major depressive disorder. Primary Psychiatry. 2008;15(11):44-47.

6. Nelson JC, Papakostas GI. Atypical antipyschotic augmentation in major depressive disorder: a meta-analysis of placebo-controlled randomized trials. Am J Pyschiatry. 2009;166(9):980-991.

7. Stahl S. Essential Psychopharmacology: The Prescriber's Guide. Cambridge, UK: Cambridge University Press; 2011.

8. Pies R. Handbook of Essential Psychopharmacology. Washington, DC: American Psychiatric Press Inc; 1998.

9. Hudziak GW. Buspirone. Philadelphia, PA: Lipincott Williams \& Wilkins; 2005.

10. Othmer E, Othmer SC. Effect of buspirone on sexual dysfunction in patients with generalized anxiety disorder. J Clin Psychiatry. 1987;48(5):201-203.

11. Duxon MS, Starr KR, Upton N. Latency to paroxetine-induced anxiolysis in the rat is reduced by co-administration of the 5-HT(1A) receptor antagonist WAY100635. Br J Pharmacol. 2000;130(7): 1713-1719.

12. Hogg S, Dalvi A. Acceleration of onset of action in schedule-induced polydipsia: combinations of SSRI and 5-HT1A and 5-HT1B receptor antagonists. Pharmacol Biochem Behav. 2004:77(1):69-75.

13. Dawson LA, Watson JM. Vilazodone: a 5-HT1A receptor agonist/ serotonin transporter inhibitor for the treatment of affective disorders. CNS Neurosci Ther. 2009;15(2):107-117.

14. Vilazodone FDA Package Insert. St Louis, MO: Forest Pharmaceuticals, Inc; 2011.

15. Sorbera LA, Rabassedi X, Silvestre J, Castaner J. Vilazodone hydrochloride: antidepressant-5-HT1A partial agonist-5-HT reuptake inhibitor. Drugs Fut. 2001;26(3):247-252.

16. Barowsky J, Schwartz TL. An evidence based approach to augmentation and combination strategies for treatment resistant depression. Psychiatry (Edgmont). 2006;3(7):42-61.

17. Trevedi MH, Fava M, Wisniewski SR, et al; STAR*D Study Team. Medication augmentation after the failure of SSRIs for depression. N Engl J Med. 2006;354(12):1243-1252.

18. Athanasiou M, Reed C, Rickels K. Vilazodone, a novel, dualacting antidepressant: current status, future promise and potential for individualized treatment of depression. Per Med. 2009;6(2): 217-224.

19. Stahl SM. Enhancing outcomes from major depression: using antidepressant combination therapies with multifunctional pharmacologic mechanisms from the initiation of treatment. CNS Spectr. 2010;15(2):79-94.
20. Stahl S. Combining antidepressant therapies from the initiation of treatment: a paradigm shift for major depression. J Clin Psychiatry. 2009;70(11):1493-1494.

21. Maes M, Meltzer H. The serotonin hypothesis of major depression. In: Bloom FE, Kupfer DJ, editors. Pyschopharmacology: The Fourth Generation of Progress. New York, NY: Raven Press; 2000:933-944.

22. Coplan JD, Wolk S, Klein D. Anxiety and the serotonin 1a receptor. In: Bloom FE, Kupfer DJ, editors. Pyschopharmacology: The Fourth Generation of Progress. New York, NY: Raven Press; 2000.

23. Pellow S, Chopin P, File E, Briley M. Validation of open:closed arm entries in an elevated plus-maze as a measure of anxiety in the rat. J Neurosci Methods. 1985;14(3):149-167.

24. Treit D. A comparison of anxiolytic and nonanxiolytic agents in the shock-probe burying test for anxiolytics. Pharmacol Biochem Behav. 1990;36(1):203-205.

25. Treit D, Menard J, Royan C. Anxiogenic stimuli in the elevated plus-maze. Pharmacol Biochem Behav. 1993;44(2):463-469.

26. Adamec R, Burton P. Effects of systemic injections of vilazodone, a selective serotonin reuptake inhibitor and serotonin $1 \mathrm{~A}$ receptor agonist, on anxiety induced by predator stress in rats. Eur J Pharmacol. 2004;504(1-2):65-77.

27. Lucki I. The forced swimming test as a model for core and component behavioral effects of antidepressant drugs. Behav Pharmacol. 1997;8(6-7):523-532.

28. Page ME, Detke MJ, Dalvi A, et al. Serotonergic mediation of the effects of fluoxetine, but not desipramine, in the rat forced swimming test. Pyschopharmacology (Berl). 1999;147(2):162-167.

29. Kehne JH, Bartoszyk GD, Greiner HE, et al. In vitro characterization of vilazodone as a dual-acting serotonin reuptake receptor and 5-HT1A receptor partial agonist. Poster presented at: 65th Annual Meeting of the Society of Biological Psychiatry Meeting; May 2010; New Orleans, LA.

30. Murck H, Antonijevic LA, Steiger A. Distinct temporal pattern of the effects of combined serotonin-re-uptake inhibitor and 5-HT1A agonist EMD 68843 on the sleep EEG in healthy men. Psychopharmacology. 2001;155(2):187-192.

31. Frampton JE. Vilazodone: in major depressive disorder. CNS Drugs. 2011;25(7):615-627.

32. Rickels K, Athanasiou M, Robinson DS, Gibertini M, Whalen H, Reed CR. Evidence for efficacy and tolerability of vilazodone in the treatment of major depressive disorder: a randomized, doubleblind, placebo-controlled trial. J Clinical Psychiatry. 2009;70(3): 326-333.

33. APA American Psychiatric Association. Diagnostic and Statistical Manual of Mental Disorders. Washington, DC: American Psychiatric Publishing; 2000.

34. Robinson DS, Kajdasz DK, Gallipoli S, et al. A 1 year open-label study assessing the safety and tolerabilty of vilazodone in patients with major depressive disorder. J Clin Pyschopharamcol. 2011;31(5):643-646.

35. Laughren TP, Gobburu J, Temple RJ, et al. Vilazodone: clinical basis for the US Food and Drug Administration's approval of a new antidepressant. J Clin Pyschiatry. 2011;72(9):1166-1173.

36. Stahl S. Stahl's Essential Psychopharmacology: Neuroscientific Basis and Practical Applications. Cambridge, UK: Cambridge University Press; 2008.

37. Grant BF, Harford TC. Comorbidity between DSM-IV alcohol use disorders and major depression: results of a national survey. Drug Alcohol Depend. 1995;39(3):197-206.

38. Markovitz PJ, Stagno SJ, Calabrese JR. Buspirone augmentation of fluoxetine in obsessive-compulsive disorder. Am J Psychiatry. 1990;147(6):798-800.

39. McDougle CJ, Goodman WK, Leckman JF, et al. Limited therapeutic effect of addition of buspirone in fluvoxamine-refractory obsessivecompulsive disorder. Am J Psychiatry. 1993;150(4):647-649.

40. Schneier FR, Jihad B, Raphael C, et al. Buspirone in social phobia. J Clin Psychopharmacol. 1993;13(4):251-256. 
41. Rabiner EA, Gunn RN, Wilkins MR, et al. Drug action at the 5-HT(1A) receptor in vivo: autoreceptor and postsynaptic receptor occupancy examined with PET and [carbonyl-(11)C]WAY-100635. Nucl Med Biol. 2000;27(5):509-513.
42. American Psychiatric Association. American Psychiatric Association Practice guideline for major depressive disorder in adults. American Psychiatric Association. Am J Psychiatry. 2010;150:1-26.

\section{Publish your work in this journal}

Neuropsychiatric Disease and Treatment is an international, peerreviewed journal of clinical therapeutics and pharmacology focusing on concise rapid reporting of clinical or pre-clinical studies on a range of neuropsychiatric and neurological disorders. This journal is indexed on PubMed Central, the 'PsycINFO' database and CAS.
The manuscript management system is completely online and includes a very quick and fair peer-review system, which is all easy to use. Visit http://www.dovepress.com/testimonials.php to read real quotes from published authors.

\footnotetext{
Submit your manuscript here: http://www.dovepress.com/neuropsychiatric-disease-and-treatment-journal
} 